\title{
Elastic modulus in the crystalline region of poly( $p$-phenylene terephthalamide)
}

\author{
R. J. Gaymans, J. Tijssen, S. Harkema and A. Bantjes \\ Department of Chemical Technology, Technological University of Twente, The Netherlands \\ (Received 13 January 1976)
}

\begin{abstract}
Fibres from aromatic polyamides have a much higher Young's modulus than fibres from aliphatic polyamides. In order to contribute to the explanation of this observed difference we looked at one of the ultimate properties, the elastic modulus in the crystalline region in the chain direction $\left(E_{\mathrm{cr} / /}\right)$. We carried out measurements on a bundle of filaments of PRD 49 fibre, which we identified by i.r. and $X$-ray analyses as poly ( $p$-phenylene terephthalamide). With the $X$-ray technique we determined

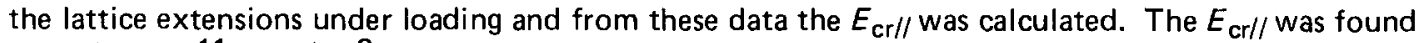
to be $20 \times 10^{11} \mathrm{dyne} / \mathrm{cm}^{2}$ which is in good agreement with the calculated $E_{\mathrm{cr} / / \text {, but not very }}$ different from that of nylon-6,6. The Young's modulus was found to be $11 \times 10^{11} \mathrm{dyne} / \mathrm{cm}^{2}$.
\end{abstract}

\section{INTRODUCTION}

Aromatic polyamides form a class of polymers which have thermal and mechanical properties that are quite different from those of the aliphatic polyamides. Fibres of aromatic polyamides have a much higher Young's modulus than corresponding aliphatic ones.

Aromatic polyamides are thought to be very good materials for reinforcing fibres ${ }^{1-4}$. The properties of these polyamides have been reviewed by Black ${ }^{4}$. He states that a possible explanation for the extraordinary properties of these polymers is due to the extended orientation rather than to the high crystallinity. Since aromatic polyamide chains are much stiffer than those of aliphatic polyamides, chain folding is less likely and a fully extended chain is obtained much easier.

Both the crystallinity and the orientation seem to be very high for these aromatic polyamide fibres. Northolt and Van Aartsen ${ }^{5}$ suggest that poly( $p$-phenylene terephthalamide) (PpPT) has a paracrystalline structure with a long order. They did not observe any periodicity up to $250 \AA$ with small-angle scattering.

Dulmage and Contois ${ }^{6}$ developed a model for the calculation of the Young's modulus from the moduli of the amorphous and crystalline phases and the degree of crystallinity, assuming the crystalline phase to be fully oriented and the amorphous phase to be unoriented.

Holliday and White ${ }^{7}$ have given a review on the present status of the factors governing the Young's modulus and the techniques available for measuring them.

One of these basic properties is the modulus in the crystalline region in the chain direction $\left(E_{\mathrm{cr} / /}\right)$. The experimentally determined values of $E_{\mathrm{cr} / /}$ agree on the whole fairly well with the calculated values.

Fielding Russell ${ }^{8}$ has calculated the $E_{\mathrm{cr} / /}$ for P $p$ PT using the method of Treloar with assumed chain dimensions and found a value of $20 \times 10^{11} \mathrm{dyne} / \mathrm{cm}^{2}$, which we also found with the now available cell dimensions ${ }^{5}$.

\section{EXPERIMENTAL}

\section{Sample preparation}

The elastic modulus was determined using an industrial fibre from Du Pont (PRD 49). In order to prepare a film for i.r. analysis the Du Pont product was dissolved in chlorosulphonic acid and precipitated in cooled methanol $\left(-60^{\circ} \mathrm{C}\right)$. A fine suspension of gelly particles was obtained by warming up under vigorous stirring. Filtration of the suspension gave a fairly homogeneous film. After washing and drying an i.r. spectrum of the film was taken, and compared with the spectra of poly ( $p$-phenylene terephthalamide) and poly $(p-$ benzamide) synthesized in our laboratory (Figure 1). As can be seen the i.r. spectra of PRD 49 and poly ( $p$-phenylene terephthalamide) are identical.

The X-ray diffraction pattern of PRD 49 fibre gave the same reflections as Northolt and van Aartsen ${ }^{5}$ obtained on PpPT. From these results and the literature data ${ }^{3}$ we assumed PRD 49 to be poly ( $p$-phenylene tere phthalamide).

One filament of the fibre was too thin to obtain strong enough reflections so a bundle of 25 filaments was used. In order to be sure that all the filaments were equally well stressed both ends of the bundle were kit together while each filament was under a preload of $1.3 \mathrm{~g}$. The ends were imbedded in such a way that easy clamping was possible. The length of the bundle of filaments between the imbedded areas was $4 \mathrm{~cm}$.

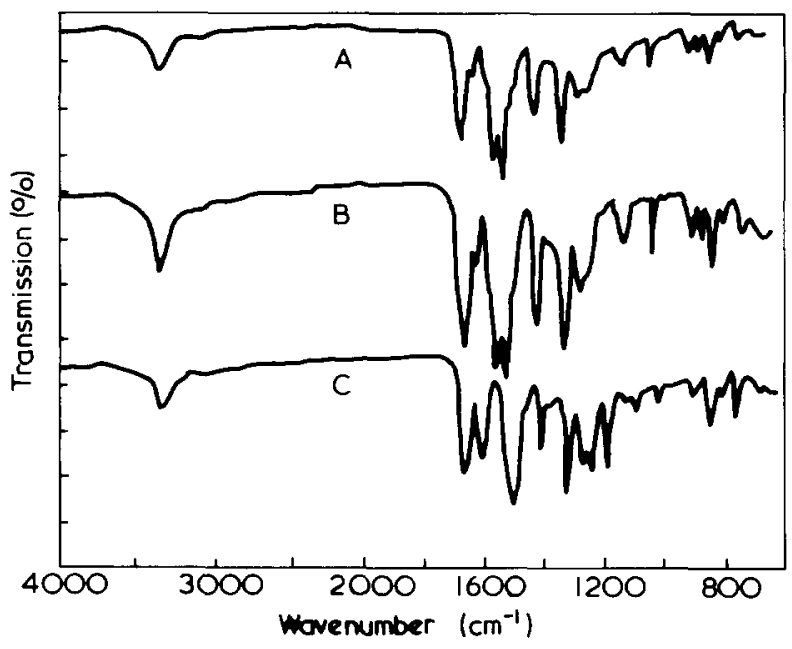

Figure 1 Infra-red spectra of polymer films of A, PRD 49; B, poly ( $p$-phenylene terephthalamide) and $C$, poly ( $p$-benzamide) 


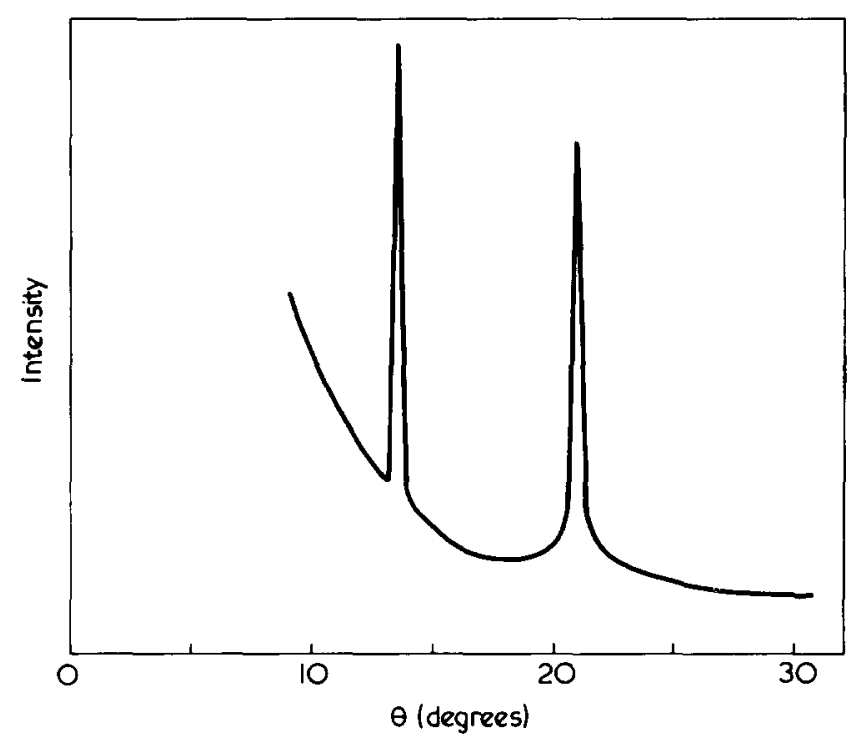

Figure $2 \mathrm{X}$-ray intensity curve in the 00/-plane

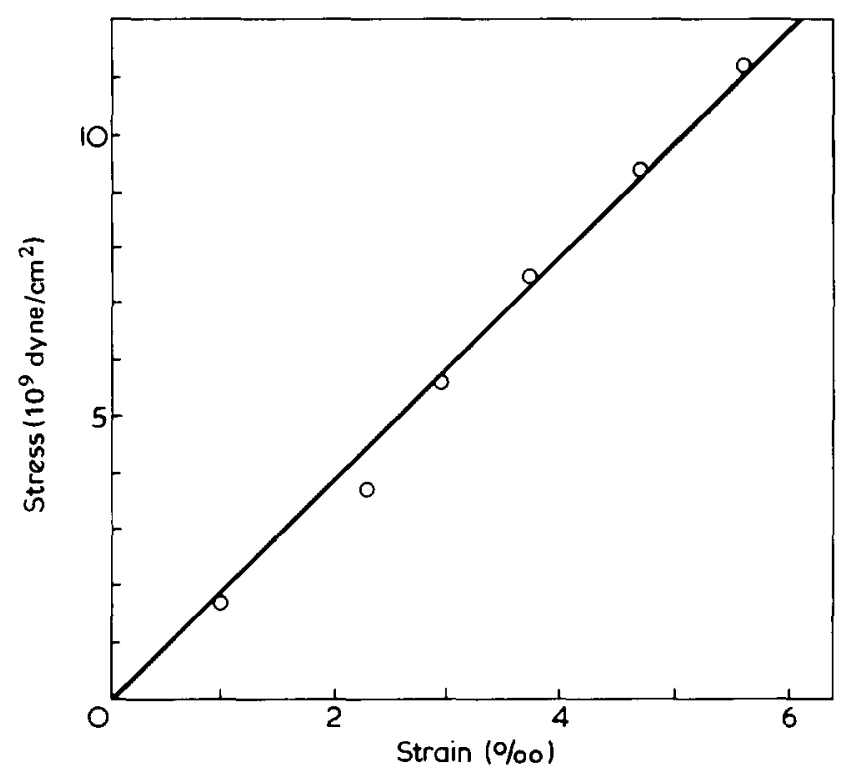

Figure 3 Applied stress versus lattice extension

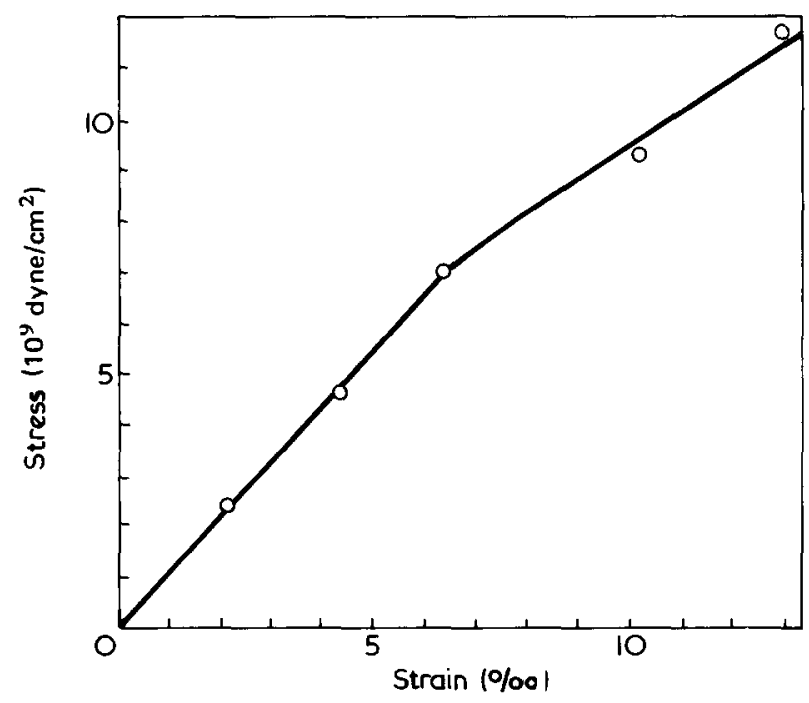

Figure 4 Applied stress versus macroscopic strain

\section{Apparatus}

The X-ray method was chosen for the determination of the $E_{\mathrm{cr} / /}$. Sakaradu ${ }^{9}$ gives a detailed set-up for determining the lattice extensions. A Nonius diffractometer with a nickel filtered $\mathrm{CuK} \alpha$-beam was used.

We measured, at any one time, an angular range of $\theta \pm 0.12^{\circ}$. Reflections were scanned by steps of $0.1^{\circ}$ with a counting time for each point of $4000 \mathrm{sec}$. From these intensities the centre of gravity of the reflection curve was calculated. The samples were loaded with dead weights and from the shifts of the gravity point the lattice extensions were calculated. Friction losses in the loading set-up were less than $5 \mathrm{~g}$. The macroscopic stress-strain curve was obtained by loading a bundle of filaments and measuring the lengthening with a kathetometer. The load was increased each hour.

\section{RESULTS AND DISCUSSION}

The crystal structure of PpPT is given by Northolt and Van Aartsen ${ }^{5}$ as monoclinic with the cell dimensions $a=$ $7.87 \AA, b=5.18 \AA, c=12.9 \AA$, and $\gamma=90^{\circ}$. The space group is $P n$ or $P 2_{1} / n$. For the determination of $E_{\mathrm{cr} / /}$ the reflections from the $00 l$-planes were measured. The normals of these planes are parallel to the chain axis. The intensity curve is measured from a bundle of filaments as a function of $\theta$ (Figure 2). In the intensity curve the reflections 004 and 006 can be seen. The reflections $00 l$ etc., where $l$ is odd, are expected extinctions. The 006 plane reflection was chosen to be measured.

It is quite important to note that in the measured region the structure factor is not very dependent on $\theta$. Therefore the reflection maximum coincides with the lattice factor maximum so that we can use the Bragg law.

The stress-lattice extension data are given in Figure 3. The $E_{\mathrm{cr}} / /$, calculated from the slope of the curve, is $20 \times$ $10^{11}$ dyne $/ \mathrm{cm}^{2}$.

The macroscopic stress-strain data are given in Figure 4. Young's modulus determined from these data is $11 \times 10^{11}$ dyne $/ \mathrm{cm}^{2}$. As can be seen the calculated and the experimental $E_{\mathrm{cr} / /}$ agree very well and these values are not very different from those obtained on nylon $-6,6^{11}$.

So the higher Young's modulus of these aromatic polyamide fibres cannot be explained by the ultimate property, the elastic modulus in the crystalline region in the chain direction.

\section{REFERENCES}

1 Rothuizen, J. H. Text. Inst. Ind. 1973, 11, 142

2 Wilfong, R. E. and Zimmerman, J. J. Appl. Polym. Sci. 1973, 17, 2039

3 Gan, L. H., Blais, P., Carlsson, D. J., Suprunchuk, T. and Wiles, D. M. J. Appl. Polym. Sci. 1975, 19,69

4 Black, L. B. J. Macromol. Sci. (A) 1973, 7, 99

5 Northolt, M. G. and van Aartsen, J. J. Polym. Lett. 1973, 11 , 333

6 Dulmage, W. J. and Contois, L. E. J. Polym. Sci. 1958, 28, 275

7 Holliday, L. and White, J. W. Pure Appl. Chem. 1971, 26, 545

8 Fielding-Russell, G. S. Text. Res. J. 1971, 41, 861

9 Sakurada, I., Nukushina, Y. and Ito, T. J. Polym. Sci. 1962, 57,651

10 Wallner, L. G. Monatsh. Chem. 1948, 79, 279

11 Dumbleton, J. H. and Buchanan, R. Polymer 1968, 9, 601 